\title{
L1 Track Triggers for ATLAS in the HL-LHC
}

\section{E. Lipeles ${ }^{a}{ }^{*}$ for the ATLAS collaboration}

${ }^{a}$ University of Pennsylvania, High Energy Physics Group, Department of Physics, 209 S. 33rd Street, Philadelphia, PA 19104, United States of America

E-mail: lipeles@hep.upenn.edu

\begin{abstract}
The HL-LHC, the planned high luminosity upgrade for the LHC, will increase the collision rate in the ATLAS detector approximately a factor of 5 beyond the luminosity for which the detectors were designed, while also increasing the number of pile-up collisions in each event by a similar factor. This means that the level-1 trigger must achieve a higher rejection factor in a more difficult environment. This presentation discusses the challenges that arise in this environment and strategies being considered by ATLAS to include information from the tracking systems in the level-1 decision. The main challenges involve reducing the data volume exported from the tracking system for which two options are under consideration: a region of interest based system and a intelligent sensor method which filters on hits likely to come from higher transverse momentum tracks.
\end{abstract}

KEYWORDS: trigger; lhc; track.

${ }^{*}$ Corresponding author. 


\section{Contents}

1. Introduction 1

1.1 The Current System 2

1.2 Motivation 2

2. L1 Tracking and Data Suppression Methods 2

2.1 Unseeded: Doublet Layer Method 3

2.2 Seeded: Region of Interest Method 4

$\begin{array}{lll}\text { 3. Summary and Comparison of Methods } & 7\end{array}$

\section{Introduction}

The HL-LHC is a planned upgrade of the LHC to a luminosity of $5 \times 10^{34} \mathrm{~cm}^{-2} \mathrm{~s}^{-1}$, which is five times the original design luminosity of the ATLAS detector and trigger. This additional luminosity increases the pressure on the trigger system because of both the higher rate and the expected pile-up of 100-200 collisions per beam crossing (depending in the bunch spacing). In order to cope with this increase in the number of particles from collisions in each beam crossing, the ATLAS tracking systems will be entirely replaced with a silicon only tracker. The readout of the new tracker will be challenging because the number of bits per event will be much larger than present. New, faster readout technology will be used to meet this challenge and achieve a readout rate, measured in events per second, similar to the present detector. Going two orders of magnitude beyond that to implement prompt readout of the full upgrade tracker is not considered feasible. This means that the rate of events accepted by first-level of the trigger, L1, cannot increase significantly over the $75 \mathrm{KHz}$ of the current system. The ATLAS baseline plan for the HL-LHC is to have $100 \mathrm{KHz}$ of available readout bandwidth.

The luminosity increase will increase the rate of interesting events (this is the purpose of the upgrade), but the pile- up increase will make it more difficult to distinguish interesting events from background. One therefore needs a trigger that is at the same time faster and more sophisticated. In Section 1.2, the motivation for a track trigger is described. Then in Sections 2.1 and 2.2, two strategies are for data reduction in the track trigger are described. Finally, the two methods are compared in terms of their impact on the detector and physics output in the Section 3 


\subsection{The Current System}

The current ATLAS trigger [1] is a three level system. The first level is implement in hardware with a decision latency (including the time to return the decision to the detect front-ends) of $2.5 \mu \mathrm{s}$. After the first level, the data is transmitted from the detector front-ends to a set of PCs called Readout Out Subsystems (ROSs) which buffer the data awaiting the second level, L2, trigger decision. The L2 trigger is implemented in software on commercial PCs connected via a network to the ROSs. Each L2 processor can request a subset of the total data in order to make decisions. This subset is generally a region around an object, e.g. $e / \gamma, \mu, \tau$ defined by the L1. These regions are referred to as Regions of Interest or ROIs. The L2 accepts 3-5 KHz of events for which the full event is then built from the data buffered in the ROSs and propagated to the third level of the trigger called the event filter, EF. Finally, the EF accepts $200-400 \mathrm{~Hz}$ for permanent storage and offline analysis. The event rates and structure of the L2 and EF for the upgrade are not yet defined, but one can take as a design parameter what physics we would like the L2 and EF to have option of selecting. That is if an event is rejected by L1 it cannot accepted by later levels.

\subsection{Motivation}

The main challenges for the level 1 trigger in the HL-LHC consist of the overall rates, the degradation of efficacy of the calorimeter isolation and missing energy trigger $E_{\mathrm{T}}^{\text {miss }}$. For the calorimeter based objects, $e / \gamma, \tau$, jets, and $E_{\mathrm{T}}^{\text {miss }}$, the trigger thresholds could just be raised, although with potentially negative impacts on the physics reach of the experiment. In particular, a reasonable precaution would be to ensure that the single lepton trigger remain efficient for accepting leptons from $W \rightarrow \ell v$ and $t \bar{t} \rightarrow \ell v j j b b$, which means $p_{\mathrm{T}}$ thresholds of order $30 \mathrm{GeV}$.

For the muon system, there is an additional challenge. Because of the limited resolution of the current level L1 muon chambers, thresholds which reach plateau efficiency above $20 \mathrm{GeV}$ continue to accept a significant number of events below $20 \mathrm{GeV}$ [2]. This means that one cannot raise the $p_{\mathrm{T}}$ requirement to remove events. The inner-detector track information from a track trigger would substantially improve the muon momentum resolution.

In addition to the rates increasing, we also anticipate efficiency of calorimeter-based isolation to degrade due to particles from other collisions entering the isolation cones. Track-based isolation can use a vertex requirement to remove the majority of the effect of other collisions making it robust against increased pile-up. Studies for the FTK project have shown this effect for lower the lower pile-up levels expected for the ATLAS Phase-I upgrade [3]. An important implication of this possible application of a track trigger is the need for precise vertexing and the reconstruction of tracks down to transverse momenta $1-2 \mathrm{GeV}$.

In addition to the track isolation and muon resolution described above, an L1-track trigger could be used for track-shower matching in electron identification and for $b$-jet tagging. Both of these algorithms are in use in the current ATLAS L2 trigger.

\section{L1 Tracking and Data Suppression Methods}

The current model of the all silicon ATLAS tracker upgrade is 4 layers of pixels detectors, 3 layers of short strips, and 2 layers of long strips, moving progressively out from the interaction point. This 


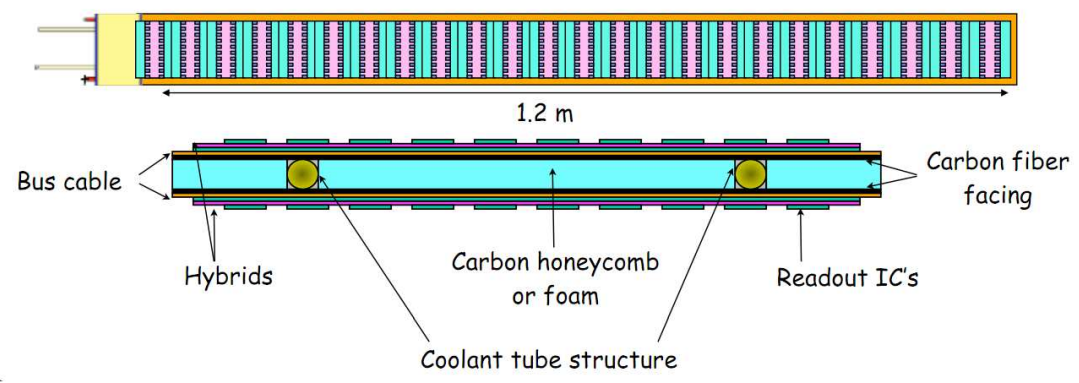

Figure 1. Stave concept for the strip detector construction using a double-sided integrated electrical, thermal, and mechanical system

amounts to approximately 400 millions pixels and 45 million strip detector channels. One possible design for the strip detectors is a double-sided integrated electrical, thermal, and mechanical system called a "stave" in which there are two sensors placed on opposite sides of a foam support with embedded cooling, shown in Figure 1. In the baseline design, the sensors on opposite-sides of the "stave" have a small stereo angle to provide full 3-d information from the combination of the two sides.

The readout bandwidth for these is expected to be equivalent to $100 \mathrm{KHz}$ of full events. Increasing the readout bandwidth would require more optical links, metal bus cables, and transmission power, and hence cooling. Increasing the readout rate to $40 \mathrm{MHz}$ in order to run full-detector tracking on each event would almost certainly require too much material degrading the offline tracking resolution. It is therefore necessary to filter the data before reading it out of the detector front-ends. We are currently considering two different filtering methods. This first possible methods [4], described in Section 2.1, filters the hits on the strip detectors before even transmitting them to the ends of the barrel "staves" or end-cap "petals". The second method, described in Section 2.2 splits the L1 trigger into an L0 and an L1 trigger, where the L0 specifies regions of the detector to be readout and used for the $\mathrm{L} 1$ decision. Track finding hardware is not discussed here but options can be found in References [5, 3].

\subsection{Unseeded: Doublet Layer Method}

The first possible data reduction method is a track $p_{\mathrm{T}}$ filter. In the solenoidal magnetic field of the ATLAS inner detector, charged particles curve in the $r-\phi$ plane. High- $p_{\mathrm{T}}$ tracks have small curvatures while low- $p_{\mathrm{T}}$ tracks have larger curvatures. The doublet method for track hit filtering relies on two axial strip layers placed close together at large radii in the tracker. As drawn in Figure 2(a), the low $-p_{\mathrm{T}}$ tracks will enter at an angle leaving hits separated in $\phi$ while high- $p_{\mathrm{T}}$ tracks leave hits that are closely spaced in $\phi$. The main source of false low $\Delta \phi$ hits is not random combinations, but correlated hits due to nuclear interactions and photon conversion, as shown in Figure 2(b). The separation in $\phi$ for $1 \mathrm{GeV}$ and $20 \mathrm{GeV}$ tracks is shown in Figure 3(a) and the effective reduction in hit rate as a function of the $\phi$ separation is shown in Figure 3(b). A data reduction of order 50 would be achievable.

The double layers could be constructed by removing the stereo angle between the strips on the opposite sides of the long strip staves which are at the radii of $80 \mathrm{~cm}$ and $100 \mathrm{~cm}$ and implementing 


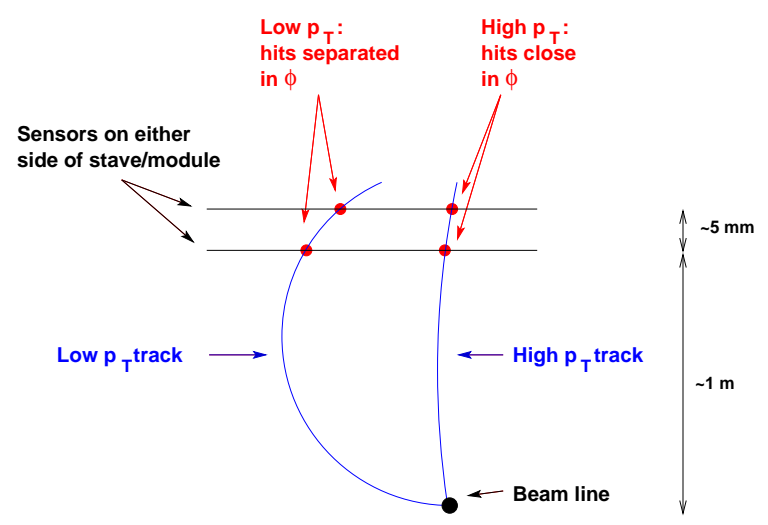

(a) Separating low- $p_{\mathrm{T}}$ and high- $p_{\mathrm{T}}$ tracks

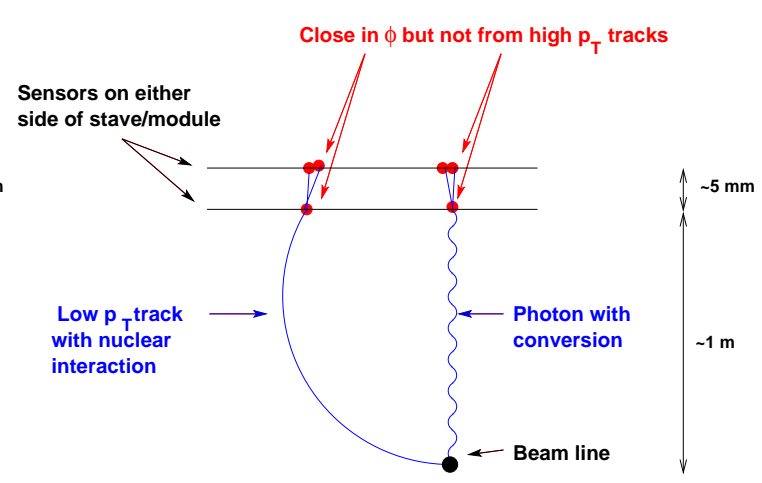

(b) Sources of false low $\Delta \phi$ hits

Figure 2. Schematic view of the separation of low and high $p_{\mathrm{T}}$ tracks using hit separation

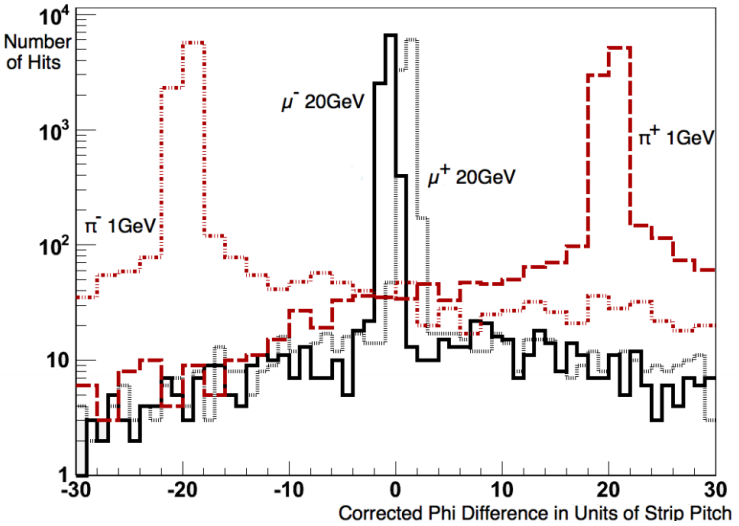

(a) Separation of low and high $p_{\mathrm{T}}$ tracks in $\Delta \phi$

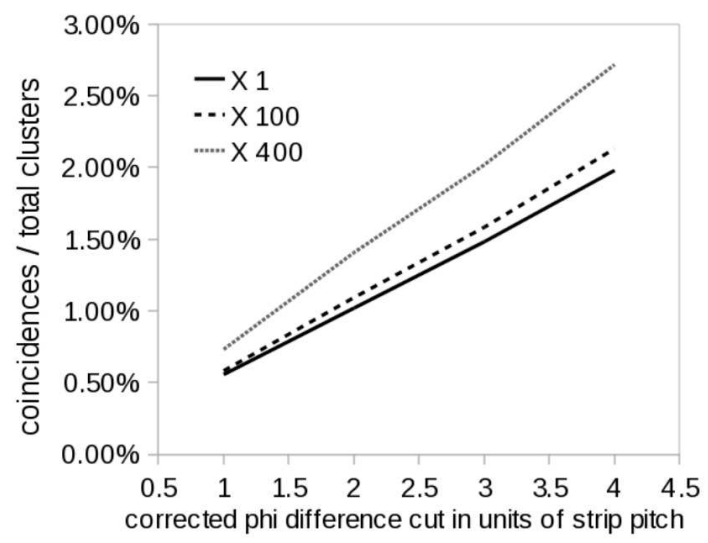

(b) Data reduction as a function of $\Delta \phi$ requirement

Figure 3. Separation of low and high $p_{\mathrm{T}}$ tracks in $\Delta \phi$ and the corresponding data reduction

a communication between them. A flexible cable could be added to the stave construction that wraps from one-side of the stave to the other. Then each sides hit information can be sent via a high-speed serial link to a correlator logic block in another chip.

Because the outer layers of the tracker have lower occupancies, there is already an excess of bandwidth relative to the inner layers. In the above example, an estimate of the required bandwidth is of order twice the $100 \mathrm{KHz}$ "natural bandwidth" that is already planned for these devices, which is considered manageable. The readout of the matched hits would be synchronous requiring a small latency. Combined with a fast track finding system [5, 3], an overall L1 latency within the latency of the current ATLAS L1 trigger $(2.5 \mu s)$ could be achieved.

\subsection{Seeded: Region of Interest Method}

Another option for reducing the data rate is a regional filter. This method requires seeding. The regions to be used in the filter must be specified by an earlier level of the trigger. For this method, the L1 trigger is split into an L0 and an L1 trigger. The L0 implementation would be similar if not the same as the current ATLAS L1 trigger [1] and would specify "regions of interest", ROIs, 
In Front-End ASIC

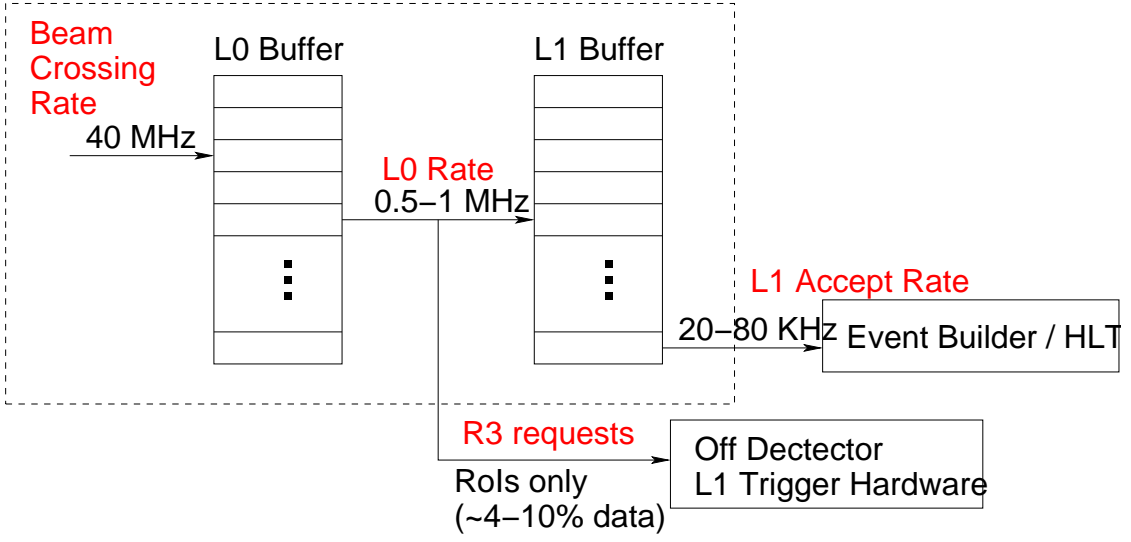

Figure 4. Front-end with support of two-buffer regional readout scheme

in which the L1 trigger would extract the hit information from the detector front-ends and do track finding.

As part of the scheme a second buffer is added to the detector front-end readout chips. The first buffer is a synchronous buffer with a $40 \mathrm{MHz}$ input similar to the current detector. After the L0 decision the data would be copied to a second buffer and await either a regional readout request or a full L1 acceptance and readout, as shown in Figure 4

For a buffer that stores a fixed number of events the available L0 latency would then be

$$
\text { L0 Latency }=\frac{\text { L0 Buffer length (in events) }}{\text { Beam Crossing rate }}=\frac{128}{40 \mathrm{MHz}} \approx 3.2 \mu \mathrm{s},
$$

where a example buffer length of 128 events is shown. For the same buffer length, the available L1 latency is then larger by the ratio of the beam-crossing to $\mathrm{L} 0$ accept rates

$$
\text { L1 Latency }=\frac{\text { L1 Buffer length (in events) }}{\text { L0 Rate }} \approx \frac{128}{500 \mathrm{KHz}} \approx 256 \mu \mathrm{s},
$$

where an possible L0 accept rate of $500 \mathrm{KHz}$ is shown. Notice that a very long L1 latency can be accommodated with a relatively small buffer, because events going into the second buffer are preselected. Finally the bandwidth required can be calculated as

$$
\begin{aligned}
\text { Bandwidth } & =\mathrm{L} 1 \mathrm{Rate}+\mathrm{L} 0 \mathrm{Rate} \times \text { fraction of data in RoIs } \\
& \approx 50 \mathrm{KHz}+500 \mathrm{KHz} \times 10 \% \approx 100 \mathrm{KHz}
\end{aligned}
$$

where the region readout has been assumed to amount to up to $10 \%$ of the detector volume.

This system has a more complex logical structure but does not appreciably change the geometry or data bandwidth for the new tracker. The overall scheme is diagrammed in Figure 5. The sequence of events is a follows:

1. Beam crossing

2. After $2.5 \mu$ s the L0 system selects of order $500 \mathrm{KHz}$ for region readout 


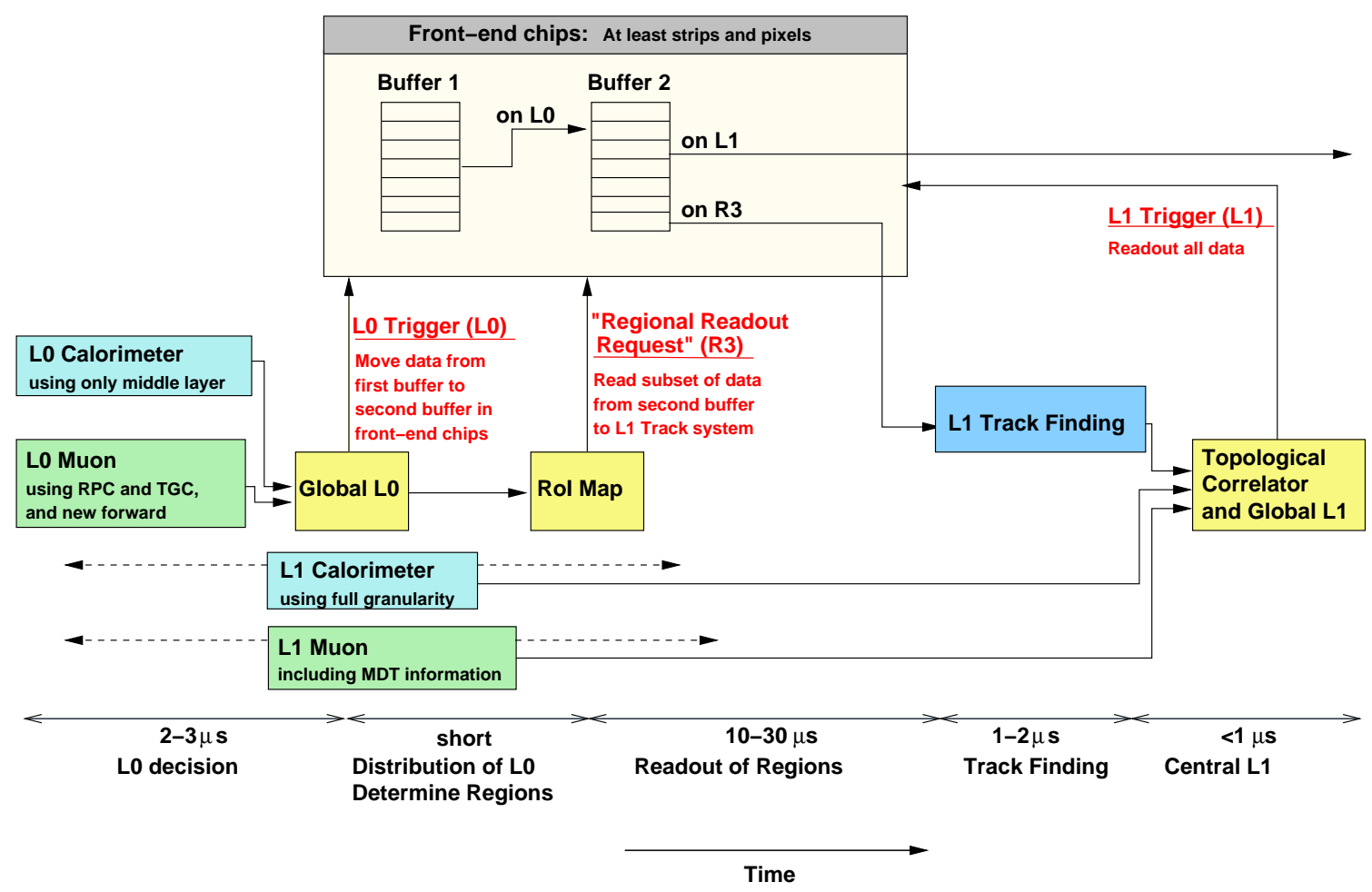

Figure 5. Time-line for a two-buffer regional readout system including the possible L1 muon and calorimeter triggers in addition to the L1 track trigger

3. Sometime less than $\mathscr{O}(10) \mu$ s later, the L0 system specifies the regions, up to approximately $10 \%$ of the detector volume, in which to readout tracking information. The corresponding modules are sent "regional readout requests".

4. The data is extracted from the front-end chips in 10-30 $\mu \mathrm{s}$. Since the baseline design expects to readout the full detector at $100 \mathrm{KHz}$, the mean time to readout a module cannot exceed 10 $\mu s$. An additional factor due to buffering for the asynchronous readout is included here.

5. A track finding algorithm is run

6. The tracking information is combined with other detector information for a final L1 decision.

The long L1 latency in this scheme has two important consequences. First it is not compatible with any of the current ATLAS detector electronics. Almost all the electronics in the system are expected to be upgrade for other reasons, however there are some electronics which are not currently planned to be upgraded and are difficult to reach. The second implication of the long latency is that other subsystems, in addition to the tracker, could also take advantage of the longer latency to use more information in the trigger. For example, the precision muon chambers (monitored drift tubes, MDTs) which have a long drift time could be used in the L1 decision. Another possibility is to use the finer granularity of the liquid argon calorimeter in combination with the tracking information to further improve the electron and photon identification. These are indicated on the time-line in Figure 5 as L1 Muon and L1 Calorimeter. 


\section{Summary and Comparison of Methods}

These two methods, unseeded doublet triggering and seeded region of interest triggering, provided significantly different handles for the suppression of backgrounds. The unseeded method provides only high- $p_{\mathrm{T}}$ tracks at at the full $40 \mathrm{MHz}$ beam-crossing rate, while the seeded methods provides all (most) momenta tracks with precision vertexing, but only in selected regions of selected events. These differences are significant in their effect on the potential scientific output of the experiment and should be considered along with the technical issues in selecting an option.

The unseeded method would fit within the current L1 latency, but would require some changes in the detector geometry âE $\breve{S}_{\text {s }}$ but imposes constraints on the geometry of the upgraded tracker. Specifically at least some of the stereo layers in the barrel would have to be changed to axial. The seeded method does not make requirements on the tracker design, but does impose on all detector components a more complex buffering scheme. Additionally, the large latency in the seeded method allows for the use additional information from other sub-detectors (muon system and calorimeter) at the $\mathrm{L} 1$ level.

\section{References}

[1] G. Aad, et. al., The ATLAS Experiment at the CERN Large Hadron Collider, 2008 JINST 3 S08004.

[2] J. Dubbert, S. Horvat, O. Kortner, H. Kroha, and R. Richter, Upgrade of the ATLAS Muon Trigger for the SLHC, 2010 JINST 5 C12016.

[3] A. Andreani, et. al., The Fast Track real time processor and its impact on muon isolation, tau and b-jet online selections at ATLAS, Proceedings of 17th IEEE-NPSS Real Time Conference (2010).

[4] M. Garcia-Sciveres, M. Gilchriese, C. Haber, B. Heinemann, T. Mueller, System concepts for doublet tracking layers, 2010 JINST 5 C10001.

[5] S. Schmitt and A. Schöning, A Fast Track Trigger for ATLAS at the Super-LHC, 2010 JINST 5 C07013. 